\title{
Functionally induced changes in water transport in the proximal tubule segment of rat kidneys
}

This article was published in the following Dove Press journal: International Journal of Nephrology and Renovascular Disease 13 April 201 I

Number of times this article has been viewed

\author{
Poul Faarup' \\ Niels-Henrik \\ Holstein-Rathlou' \\ Tove Nørgaard ${ }^{2}$ \\ Adrian Paul Harrison ${ }^{3}$ \\ Lone Bastholm' \\ Lisbeth Thatt' \\ Flemming F Johansen' \\ Viktor Hegedüs' \\ 'Department of Biomedical \\ Sciences, Panum Institute University \\ of Copenhagen, ${ }^{2}$ Department \\ of Pathology, Hillerød Hospital, \\ ${ }^{3}$ Department of Animal and Veterinary \\ Basic Sciences, Faculty of Life \\ Sciences, Copenhagen University, \\ Denmark
}

Correspondence: Adrian Harrison Section for Biochemistry, Physiology, and Nutrition, Department of Animal and Veterinary Basic Sciences,

Faculty of Life Sciences,

University of Copenhagen,

Grønnegaardsvej 7, 1870 Frederiksberg C, Denmark

Tel +4535332568

Fax +4535332525

Email adh@life.ku.dk

\begin{abstract}
To eliminate freezing artifacts in the proximal tubule cells, two cryotechniques were applied to normal rat kidneys, ie, freeze substitution and special freeze drying. In addition, salt depletion and salt loading were applied to groups of rats to evaluate whether the segmental structure of the proximal tubule could be altered. In the superficial part of the renal cortex of normal kidneys, the typical first segment structure in the proximal tubule was generally present in the early postglomerular fraction of the tubule. However, in the second segment, a special cellular phenomenon was constantly present, comprising a significant intercellular space that was easily identified using a light microscope. In the third segment, in which the presence of basolateral interdigitations is minimal, the small lateral space, which was found to be present in cryopreparations between neighboring cells from the normal kidney, was found to be enlarged by heavy salt loading of short duration. It is concluded that these cryotechniques demonstrate quantitative structural variations between superficial and deep nephrons, as well as the presence of extracellular areas between the cells of the second and the third segment, representing a structural background for the essential transport of water from the proximal tubules to the peritubular capillaries.
\end{abstract}

Keywords: proximal tubule, salt load, salt depletion

\section{Introduction}

Since the identification of the segmental structure of the renal proximal tubule approximately 40 years ago, ${ }^{1}$ only a few reports have documented the consequences associated with histological variability of these structures with regard to their functional activity. In particular, very little is known about essential transcellular water transport occurring in this part of the nephron.

It is well known that any chance of studying the mechanism of fluid transport in or among cells in the kidney may be significantly obstructed or affected by, eg, rapid postmortem changes, fixative-induced structural alterations, or perturbations caused by instrumental devices. Even the smallest of structural changes can be of significance when investigating the functional structure of a tissue, particularly when such elucidation is with the aid of a microscope. The identification of water-selective aquaporin channels in the proximal tubules of the kidney ${ }^{2-4}$ serves as an example of results based on unorthodox technical methodology. It is for this reason that a more appropriate cryotechnology approach needs to be sought and used in an attempt to minimize early postmortem artifacts ${ }^{5,6}$ and the inevitable fixation-induced changes that occur in terms of cellular morphological details. ${ }^{7}$ 
Both structural and functional differences are known to exist between $\mathrm{S} 1$ and $\mathrm{S} 2$ cell types in the proximal tubule of the kidney. Most notably, the reabsorption of glucose from the tubular fluid mainly occurs in $\mathrm{S} 1$ cells, where more than $90 \%$ of the glucose present in the lumen is reabsorbed. ${ }^{8}$ In a similar fashion, glucose, galactose, and amino acids accumulate in an osmotically active form within the small intestinal cells during reabsorption from the ileum, and this is accompanied by an increase in water content and swelling. ${ }^{9-11}$ This then forms a parallel with the S1-like cells of the proximal tubule. Another difference that exists between the initial and the final fraction of the convoluted proximal tubule is the presence of a countercurrent system, which is found between the convoluted proximal tubule and the peritubular capillaries. ${ }^{12-14}$ In this way, the oncotic pressure in the peritubular capillaries is highest at the second segment, which is important for the transmural transport of water. ${ }^{4}$

It was the aim of this study to refine and apply a more appropriate cryotechnology approach to the investigation of the renal proximal tubule structure, and to use this technique subsequently to investigate the varied structural characteristics of superficial and deep nephrons, as well as those of the first, second, and third tubular segments, arising in part from changes in salt loading. Particular emphasis is placed on the structural effects associated with high-speed cellular water transport in the rat kidney.

\section{Methods}

Our experiments were conducted in accordance with the ethical guidelines of the Animal Behavior Society and national legislation, and complied with previously published standards and advice. ${ }^{15}$

\section{Normal rats}

Eight male Wistar rats (200-250 g body weight) were allowed free access to distilled water and standard food (Altromin standard rat diet C-1035, Brogaarden, Denmark) containing $0.9 \% \mathrm{NaCl}$ (dry weight). The urine was analyzed routinely at the end of each experiment using a standard urine strip (Multistix 10 SG, Bayer Diagnostics, Bridgend, UK), and no signs of proteinuria or blood in the urine were noted. On this basis, no ischemic lesions were deemed to be present. Animals were anesthetized intraperitoneally using sodium barbiturate $35 \mathrm{mg} / \mathrm{kg}$ body weight with a reflex test to assess that an adequate level of anesthesia had been attained. The right kidney of each rat was then rapidly removed and frozen in isopentane cooled by liquid nitrogen to $-165^{\circ} \mathrm{C}$. The frozen kidneys were subsequently divided into two groups, each comprising four kidneys. The animals were then humanely killed by an overdose of anesthetic followed by neck dislocation.

\section{Group IA}

The right kidneys from four rats were freeze substituted in ethyl alcohol at $-20^{\circ} \mathrm{C}$ for 4 weeks, and thereafter transferred to $4 \%$ paraformaldehyde for fixation at room temperature for 24 hours before finally being conventionally embedded in paraffin. ${ }^{5,7}$ Sections measuring 3-5 $\mu \mathrm{m}$ in thickness were periodic acid Schiff(PAS) stained. Part of the kidney cortex was then embedded in araldite. Semithin sections of $0.5-1.0 \mu \mathrm{m}$ in thickness were stained with toluidine blue. Because this fixation procedure is not suitable for electron microscopy, an ultrastructural investigation was not performed.

\section{Group IB}

In four rats, $1.0 \mathrm{~mL}$ of a $1 \%$ solution of methylene blue in physiological saline was injected intravenously 1 second prior to the rapid removal and freezing of the right kidney. Frozen sections, $5 \mu \mathrm{m}$ in thickness, were freeze dried for 24 hours in a desiccator containing phosphorus pentoxide at $-20^{\circ} \mathrm{C}^{7}$ Thereafter, the sections were mounted in paraffin oil, thereby omitting fixation, contamination with water, and mounting chemicals (eg, xylene and mounting medium).

\section{Salt-depleted or salt-loaded rats}

A different $\mathrm{NaCl}$ diet was allocated to a total of 24 male Wistar rats (200-250 g body weight) as follows.

\section{Group 2A}

Four rats received a diet containing $0.1 \%$ (dry weight) $\mathrm{NaCl}$ (Altromin C-1036, Brogaarden, Denmark) and distilled water for 4 weeks. The animals were anesthetized intraperitoneally using sodium barbiturate $35 \mathrm{mg} / \mathrm{kg}$ body weight with a reflex test to determine that an adequate level of anesthesia had been attained. Thereafter, the right kidneys were freeze substituted and prepared as described previously. The animals were humanely killed by an overdose of anesthetic followed by neck dislocation.

\section{Group 2B}

Four rats received a diet containing $5 \%$ (dry weight) $\mathrm{NaCl}$ (Altromin C-1051, Brogaarden, Denmark) and distilled water for 4 weeks. The animals were anesthetized intraperitoneally using sodium barbiturate $35 \mathrm{mg} / \mathrm{kg}$ body weight with a reflex test to assess that an adequate level of anesthesia had been attained. Thereafter, the right kidneys were freeze substituted as described previously. The animals were humanely killed by an overdose of anesthetic followed by neck dislocation. 


\section{Groups 2C-F}

All animals in Groups 2C, 2D, 2E, and 2F were prepared as follows. After intraperitoneal anesthesia with inactin $100 \mathrm{mg} / \mathrm{kg}$ body weight, the rats were placed on a temperature-regulated table for the duration of the experiment, and a regular reflex test was performed to assess that an adequate level of anesthesia was being maintained. For each animal, a solution of $0.5 \mathrm{~g} \mathrm{NaCl}$ in $3 \mathrm{~mL}$ of distilled water $(2.85 \mathrm{M})$ was infused over a period of an hour via the femoral vein.

In four rats, the right kidney was freeze substituted immediately after the infusion of the $2.85 \mathrm{M} \mathrm{NaCl}$ solution (Group 2C). Groups 2D, 2E, and 2F comprised the controls and included four rats each, which were treated in the same manner as the animals in Group 2C. The right kidneys were removed after the intravenous infusion as follows: Group 2D after 48 hours; Group 2E after 2 weeks; and Group 2F after 4 weeks. The animals were humanely killed by means of an overdose of anesthetic followed by neck dislocation.

\section{Tissue preparation and evaluation}

After freeze substitution and PAS staining of the histological sections, Groups $1 \mathrm{~A}$ and $2 \mathrm{~A}-2 \mathrm{~F}$ were investigated quantitatively as follows. The immediate postglomerular fraction of the proximal convoluted tubule (minimal length twice that of the tubular diameter) was photographed for both superficial and juxtamedullary nephrons. On average, 15 randomly chosen nephrons from each location (superficial versus juxtamedullary) in each individual kidney were investigated. The appearance of the immediate postglomerular fraction was classified according to the following three arbitrary groupings:

- For fractions presenting with a typical cellular structure comprising high brush borders, tall cells, and a reduced luminal diameter of the tubule, as previously described, ${ }^{1}$ the term S1 was assigned.

- For fractions presenting with a mixture of cells comprising typical S1 cells intermingled with cells having structural characteristics typical of the second segment, the term S1-2 was assigned.

- For fractions presenting with cells with a low brush border, a low cellular height in tubules, and an enlarged luminal diameter, the term S2 was assigned. These cells systematically contained light microscopically identifiable basolateral spaces between the cellular interdigitations. Otherwise, the cellular structure was identical to that of S2, as described elsewhere. ${ }^{1}$

The quantitative evaluation according to this classification was made by two of the investigators (TN and $\mathrm{VH}$ ) independently of each other and in a blinded fashion. The results were compared and analyzed for statistical significance by a third investigator (NHHR).

A similar procedure was applied for evaluation of the tubular segmental loops $\mathrm{S} 1, \mathrm{~S} 1-2$, and $\mathrm{S} 2$, in the total renal cortex. In each kidney, 15 randomly chosen superficial and juxtamedullary cortical areas, each covering an area of $0.3 \times 0.2 \mathrm{~mm}$, respectively, were photographed to enable further quantitative evaluation of the loops of the proximal convoluted tubule.

In addition, a description of the structure of the third segment cells (S3) was given, because they took on a special appearance depending on the preparations used. Tissue from the contralateral kidney, lungs, liver, and spleen were taken from each rat and were conventionally prepared for microscopy analysis in order to ensure the absence of irrelevant pathological changes.

\section{Statistical analysis}

Data, which were normally distributed and of equal variance, are presented as mean \pm standard error of the mean. Statistical evaluation of the observations was made using an analysis of variance for repeated measurements. Where significant differences between the means were indicated, the individual means were compared using a Newman-Keuls test. A $P$ value of less than 0.05 was considered statistically significant.

\section{Results \\ General appearance of proximal tubule in normal kidneys}

The cellular structure of the proximal tubule in the cryopreparations of normal kidneys was consistently different from the morphology seen in similar specimens, for which no cryotechnique was employed during tissue preparation. In S1, represented by the immediate postglomerular tubular neck region, the tall cells, with a high brush border and a small tubular lumen, were present preferentially in the superficially located nephrons (see later parts of this section concerning the quantitative evaluation of cell types in the differently located nephrons, and Figures 1 and 2). However, in the juxtamedullary nephrons, S2-like tubular cells replaced the typical S1 cellular structure in the postglomerular neck region. Moreover, it is essential to note here that the structure of the segmentally identical tubular cells should be similar for the two types of cryopreparations applied, ie, the freeze-substitution technique, compared with the freeze-drying technique (Figures 1-3). 


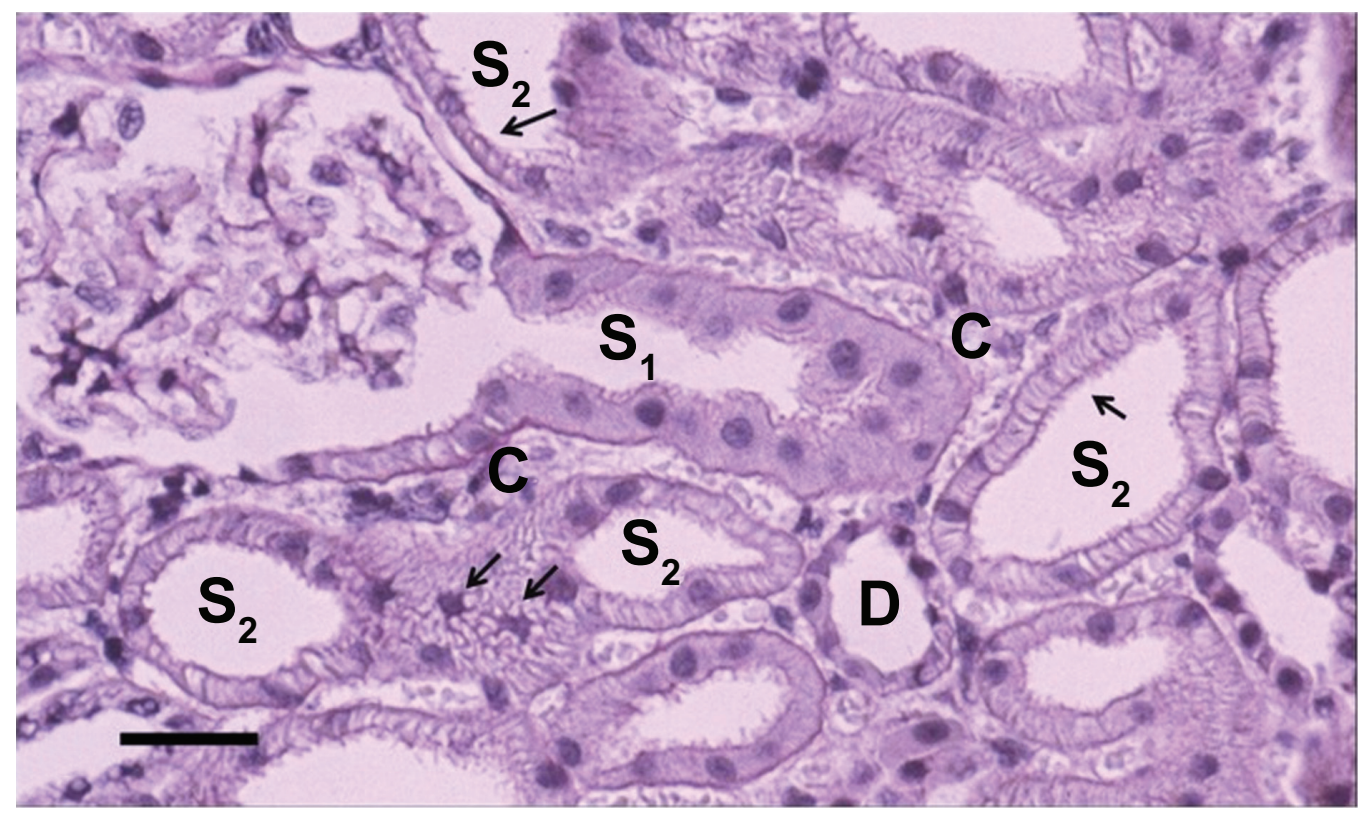

Figure I A PAS-stained, freeze-substituted normal kidney, showing the superficial cortex.

Notes: Please note that in the initial fraction of the SI segment starting from the glomerulus the cytoplasm of the SI cells has a finely granular cytoplasm, lacking a radial striation, which is typical for $\mathrm{SI}$ cells. In these tall cells the nuclei are round and the brush border is high. In the $\mathrm{S}$ segment loops the cells are shorter, however, there is variation in cellular height in the different loops (see arrows). The cells also have a reduced height of the brush border. In all S2 segments the radial cytoplasmic striation demonstrates dilated, basolateral spaces (cf. Figure 2 and 3). Contrary to the round nuclei of SI cells, here the nuclei may appear gear-wheel like, apparently a phenomenon secondary to the dilated basolateral spaces (see double arrow). Around the glomerulus a capsular space can be seen, this is always found with optimal cryo-preparations of normal kidneys. D represents the distal tubule, $C$ denotes a capillary. The scale bar represents $25 \mu \mathrm{m}$.

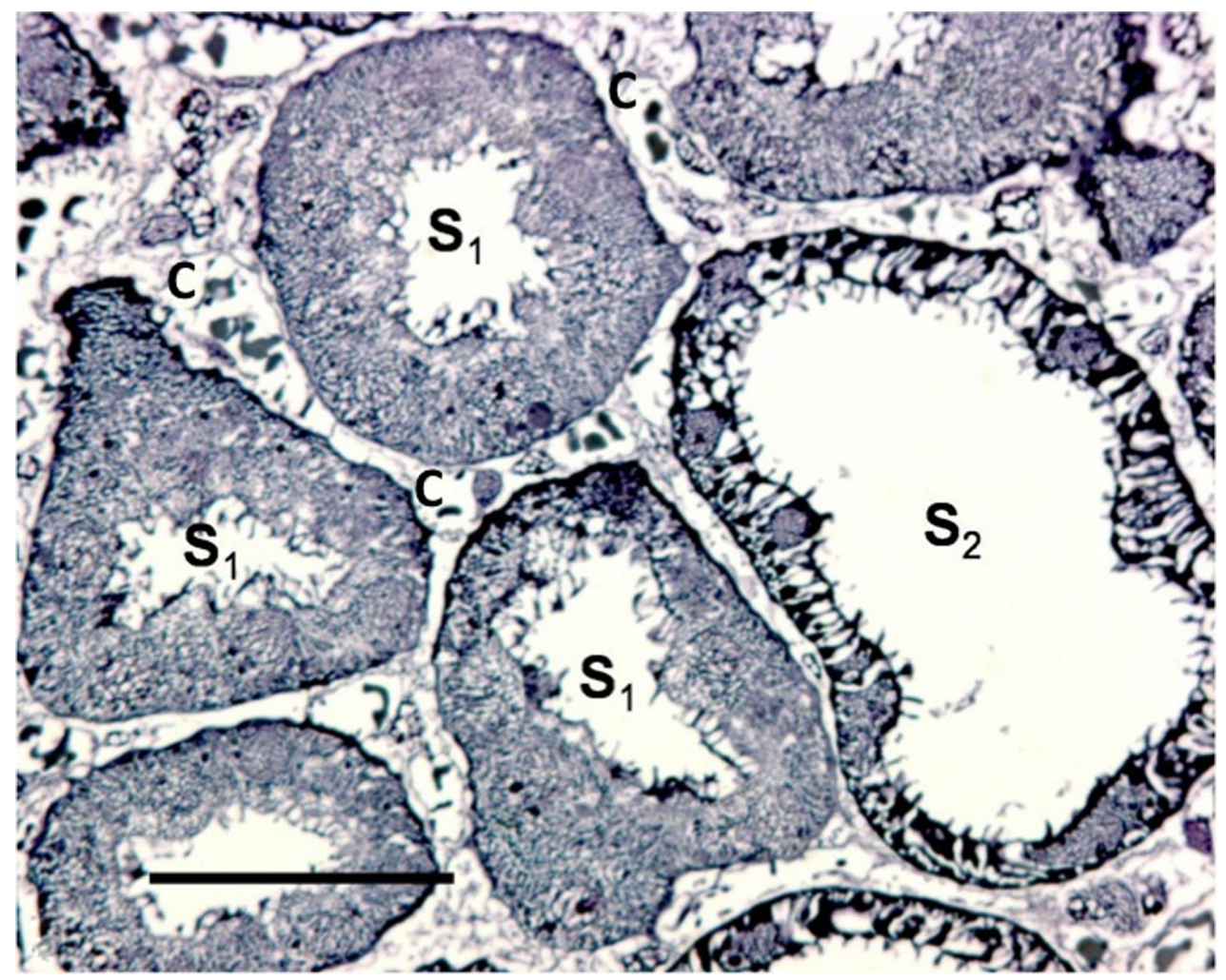

Figure 2 A freeze-substituted normal kidney, showing the superficial cortex.

Notes: Please note in this toluidin blue stained, semi-thin specimen from plastic-embedded tissue, the typical differences between the SI and S2 cells concerning diameter of the lumen and cellular height as well as the very dilated basolateral spaces between the $\mathrm{S} 2$ cells. C denotes a capillary. 


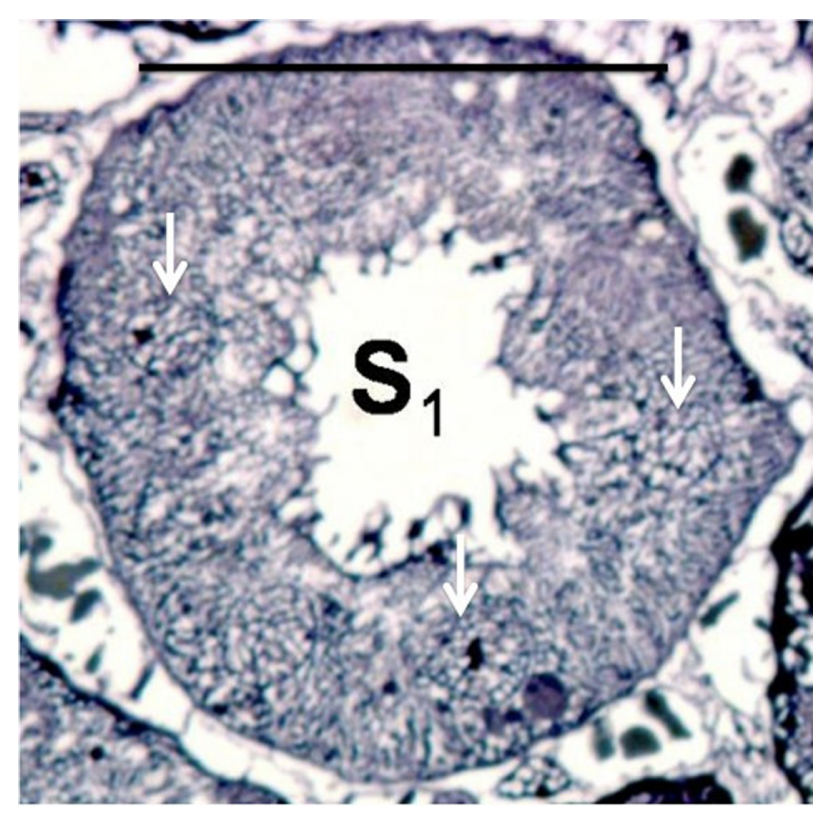

Figure 2B An enlarged region of Figure 2a. Please note the net-like structure in the nuclear content connecting the nucleolus to the nuclear membrane of the SI cell (see arrows). The scale bar represents $25 \mu \mathrm{m}$.

The most important structural difference found in both types of cryopreparations was the consistent presence of an essential extracellular space between the basolateral membranes of typical S2-like cells of the proximal convoluted tubule. Although the cell nuclei of the S1 and S3 cells were always oval or circular in shape, the S2 cell nuclei were frequently angular in appearance, presumably as a consequence of the dilated intercellular space found between the S2 cells (Figures 1, 2, and 4). In the nuclei of the proximal tubule, a special structural phenomenon could be observed, most easily seen in S1-like cells. Here, the nuclear content demonstrated a ramified network, typically connecting the nucleolus to the nuclear membrane (Figure 2B). A similar nuclear network was sometimes found in the nuclei of the endothelial cells of the peritubular capillaries.

In the proximal tubular loops located in the immediate subcapsular area there was a predominance of S2 segments (Figures 4A and 4B). In this area, S2 tubular cells constituted the dominant part of the proximal tubular loops. However, it was frequently observed that the cells of the S2 tubule lying adjacent to the capsule were lacking the dilated basolateral spaces that were found to exist between the other typical S2 segment cells. In addition to these changes, the immediate subcapsular cells were found to be structurally identical to the usual S2 segment cells in their appearance (low cell height, low brush border). The reverse of these structural phenomena was never observed in this location, and the intercellular space between the $\mathrm{S} 2$ cells of the proximal tubule, identifiable at the light microscope level, was always present outside the immediate subcapsular region.

In terms of a quantitative analysis of the cells in the juxtamedullary region, ie, in the immediate postglomerular neck

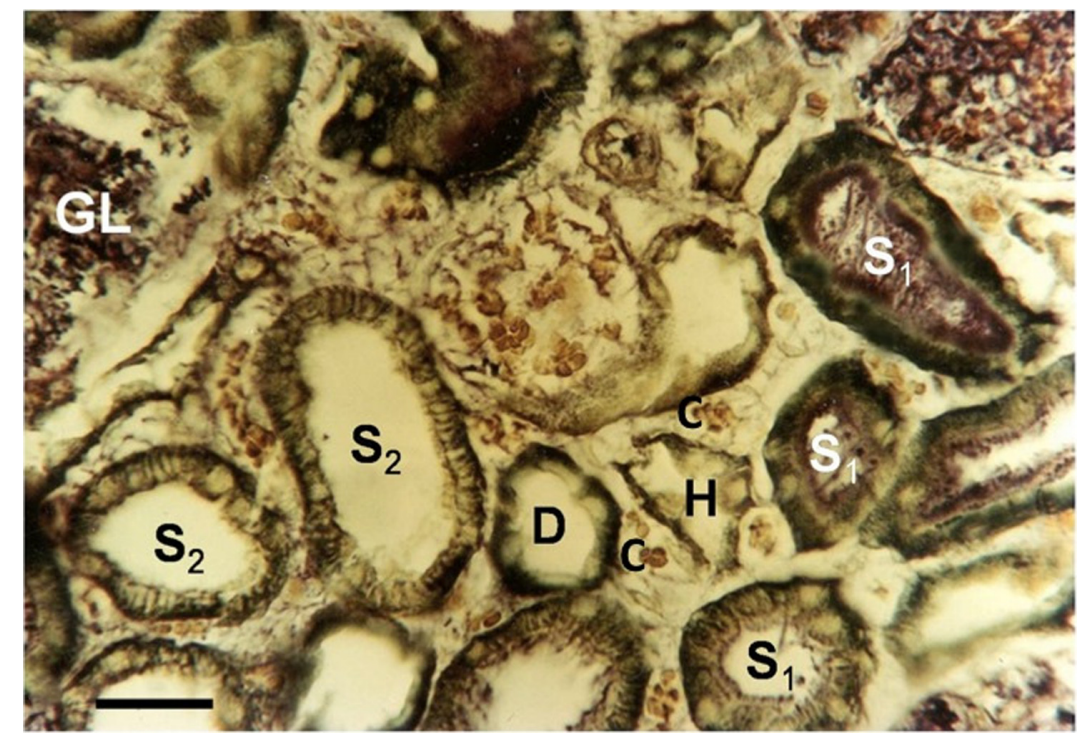

Figure 3 A freeze-dried normal kidney, vitally stained using methylene blue revealing the superficial cortex.

Notes: The lumen of some SI segments were stained by the intravenously injected methylene blue, which filtered through the glomerulus, whereas other SI loops and later tubular fractions of the nephrons do not contain any stain in the lumen. Their tissue is only stained from the capillary side. Only in the S2 segments can a radial, cytoplasmic striation be seen, demonstrating a space between the basolateral membranes among S2 cells in the functioning kidney. The nuclei of the tubular cells are unstained in this preparation. D represents the distal tubule, GL denotes the glomerulus and $\mathrm{H}$ indicates the ascending branch of the loop of Henle, $\mathrm{C}$ denotes a capillary The scale bar represents $25 \mu \mathrm{m}$. 
A

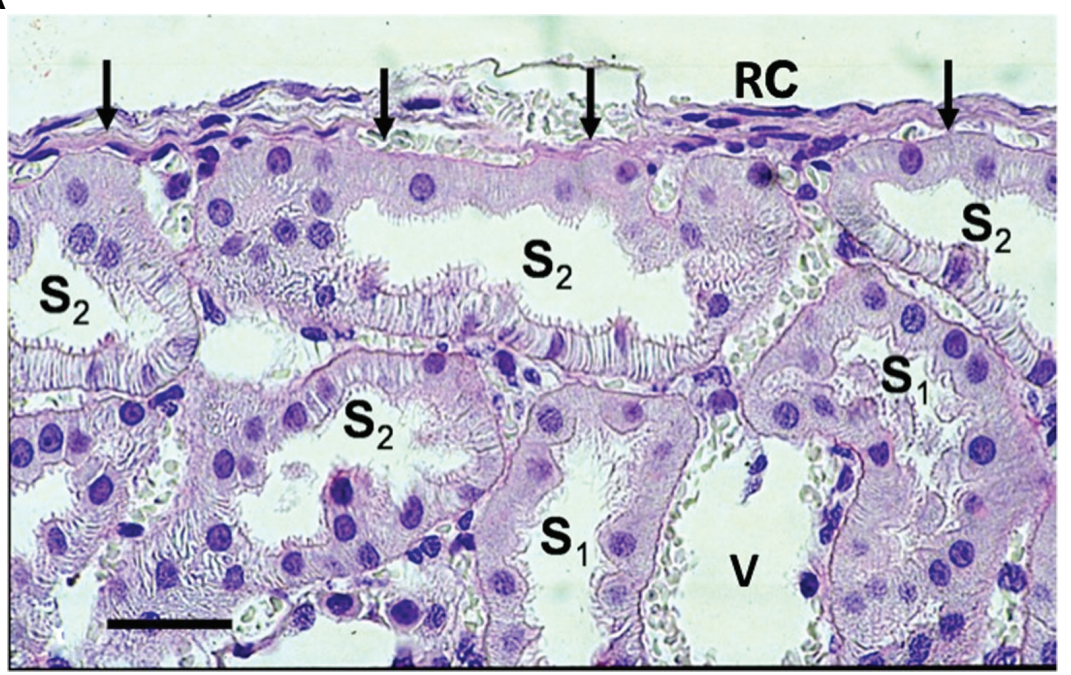

B

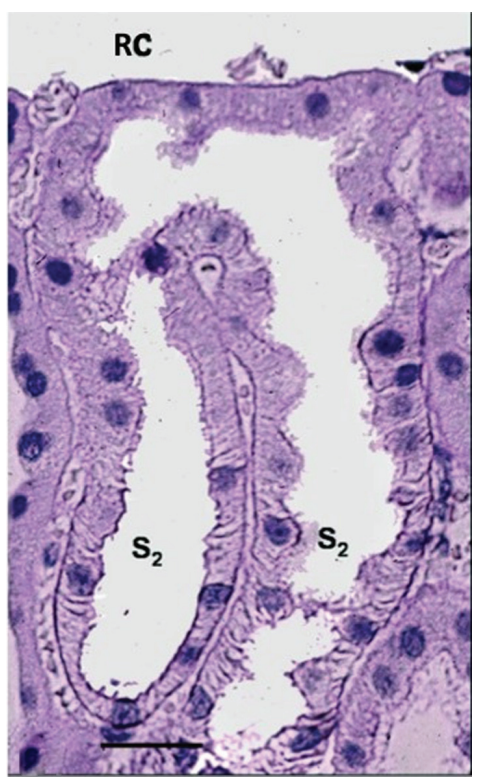

Figure 4 A PAS-stained freeze-substituted normal kidney showing the subcapsular area of the cortex.

Notes: A) The superficial fractions of the S2 segments, placed just beneath the renal capsule (RC) do not possess the usual basolateral spaces, normally seen in S2 segments (see arrows). In this segment, the angularly shaped, or gear-wheel like nuclei can only be seen in areas where the intercellular basolateral space is present. $V=$ a Venole located between two SI segment loops, the location of which is consistent with the previously described tubulo-vascular counter-current flow in the proximal convoluted tubule (Faarup et $\mathrm{al}^{13}$ ). RC denotes the renal capsule. B) A PAS-stained, freeze-substituted kidney showing a S2 tubular loop, located just beneath the renal capsule (RC). The fraction of the loop that is in contact with the capsule is lacking a basolateral interstitial space between the cells, although this is to be found both before as well as after the region of capsular contact. RC denotes the renal capsule. The scale bar represents $25 \mu \mathrm{m}$.

A

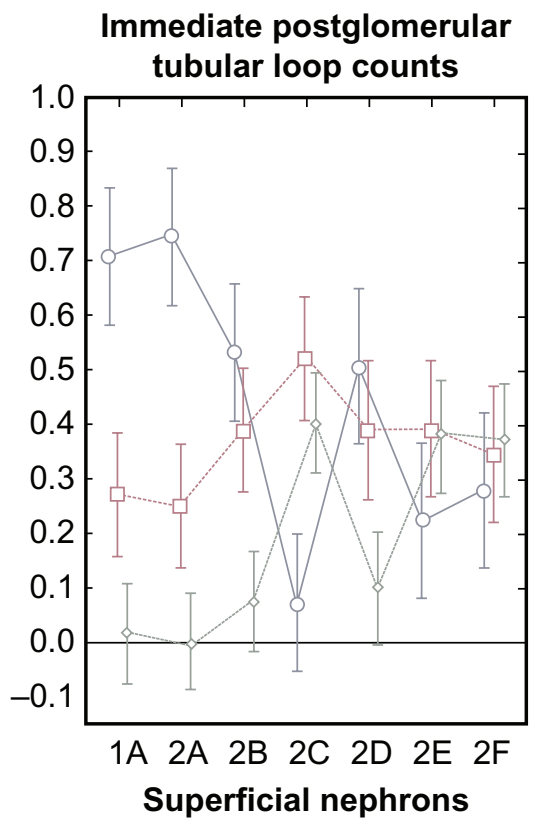

B

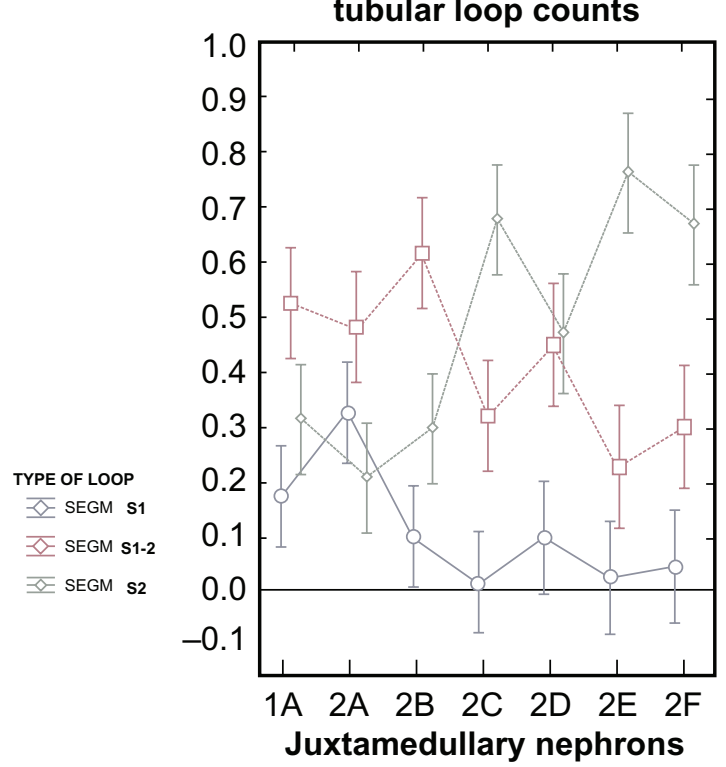

Graph I. Quantitative segmental evaluation of the immediate postglomerular fraction of the proximal convoluted tubule in freeze-substituted tissue from the renal cortex.

Notes: IA) Among the superficial nephrons from normal kidneys (Group IA) the number of SI-like cells far exceeds that of the SI-2 like cells, which are themselves significantly more frequent than the S2-like cells. Moderate salt depletion or salt loading over a period of I month (Groups 2A and 2B) does not change the distribution significantly. However, a heavy salt loading of short duration (I hour) (Group 2C) significantly reverses the frequencies of the SI, SI-2 and S2-like cells such that the presence of the SI-like structured cells falls far below the number of SI-2-like and S2-like cells in this part of the tubule. After a temporary, yet significant reversal of the structural changes in the SI- and S2-like structures some 48 hours later, the presence of both SI-, SI-2 and S2-like cells becomes nearly identical (see Groups 2D, E and F) 2 days, 2 weeks or 4 weeks after the acute intervention of the heavy salt loading. IB) In the juxtamedullary located nephrons from freeze substituted normal kidneys the presence of both the SI-like, and the S2-like cells of the immediately postglomerular located fraction of the proximal convoluted tubule were lower in number than the SI-2 like cells (Group IA). No significant changes were found during moderate salt depletion or salt loading(Groups $2 \mathrm{~A}$ and B). After a heavy salt loading of short duration (Group 2C) the SI-like cells were nearly totally eliminated, and the S2-like cells were increased in number significantly, to subsequently comprise two thirds of the structure in this region. Interestingly, even 4 weeks later the Control Group (F) did not show any change in this distribution. 

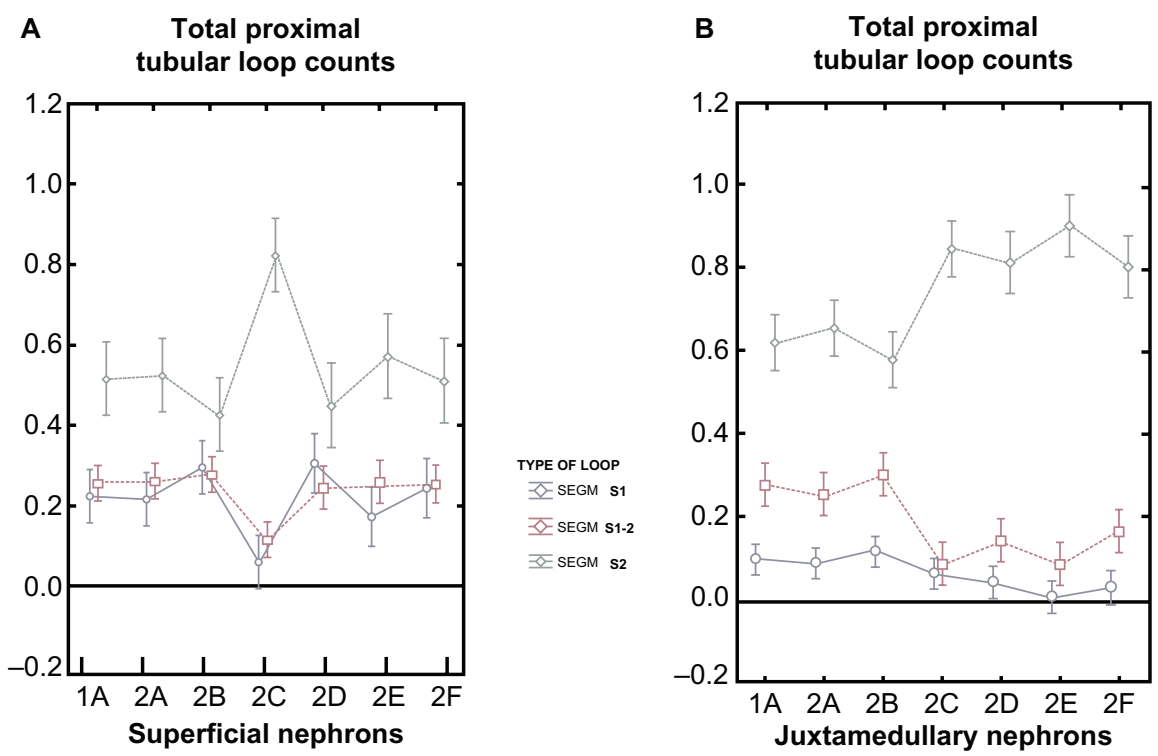

Graph 2 The percentage segmental distribution of the total loops from the proximal convoluted tubule of the renal cortex of freeze-substituted kidneys.

Notes: 2A) In the superficially located nephrons of normal kidneys (Group IA) the S2-like cells in the loops were significantly more frequent than the SI-, and SI-2 like cells, which were found to be equally frequent. Slight salt depletion or salt loading did not change these values significantly (Group $2 \mathrm{~A}$ and B). However, after a heavy salt loading of short duration (Group 2C) the frequency of S2-like cells was elevated from about $40 \%$ to more than $80 \%$. In accordance, the number of SI- and SI-2 like cells were reduced to around $10 \%$ of the total. Moreover, even 4 weeks later in the Control (Groups 2F) values were found to return to those seen in normal kidneys (see "normal" - Group IA). 2B) In juxtamedullary located nephrons in freeze-substituted kidneys the normal kidneys (Group A) revealed a number of SI-like cells that were slightly lower than those in the superficial nephrons (Graph 2A), otherwise, the distribution was rather similar. No significant changes were found with moderate salt depletion or salt loading (Groups 2A and B). However, a heavy salt loading of short duration (Group 2C) induced the number of S2-like cells to become clearly elevated in percentage terms. $\mathrm{SI}$-like and SI-2 like cells were concomitantly reduced in proportion. Notably though these structural changes did not normalize, even in the Controls up to 4 weeks later (Group 2F).

region of the normal kidney, the $\mathrm{S} 1$ segments were found to be significantly fewer in number than those measured superficially (see Graph 1A compared with Graph 1B, Group 1A). However, a total count of the convoluted proximal tubule showed a difference between superficial and juxtamedullary nephrons (significantly lower numbers of S1 segment loops) for the juxtamedullary nephrons alone (see Graph 2A compared with Graph 2B, Group 1A). In the vitally stained, freeze-dried specimens (free of fixation, water contamination, and embedding media artifact), a similar structure was observed to that of the freeze-substituted specimens with regard to the S1 and S2 loops (Figure 3).

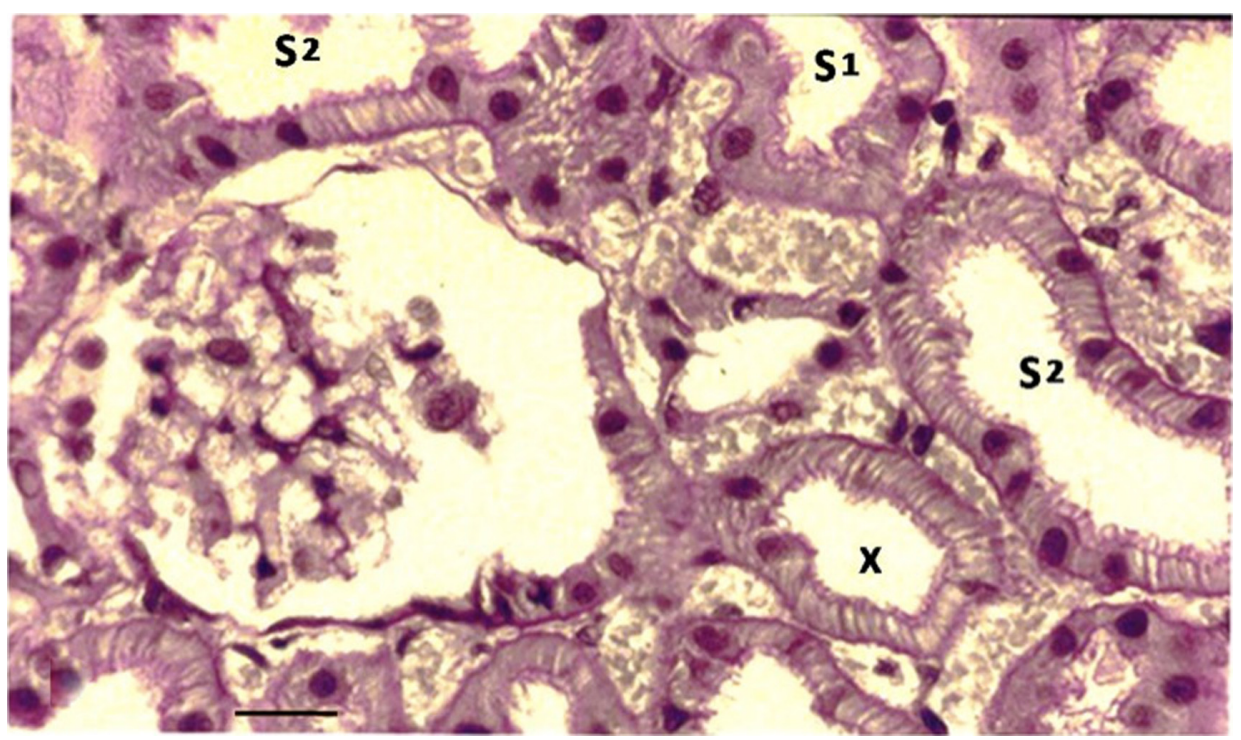

Figure 5. A PAS-stained freeze-substituted normal kidney showing a juxtamedullary nephron.

Notes: The cells in the proximal tubular loop $(\mathrm{X})$, descending from the glomerulus, have a typical S2-like structure. The scale bar represents $25 \mu \mathrm{m}$. 


\section{Kidneys from moderately salt-depleted rats}

In Group 2A, no significant differences were found in the distribution of S1, S1-2, and S2 tubular loops in the kidneys of salt-depleted rats compared with normal kidneys. This observation was valid in both the superficial and juxtamedullary regions (see Graphs 1A, 1B, 2A, and 2B).

\section{Kidneys from moderately salt-loaded rats}

In Group 2B, the quantitative data for segmentation of the proximal convoluted tubules were without significant change when compared with those in Groups 1A (normal rats) and 2A (salt-depleted rats, see Figures 1A, 1B, 2A, and 2B).

\section{Kidneys from acutely heavy salt-loaded rats and later controls}

In Groups $2 \mathrm{C}-\mathrm{F}$, when a heavy salt loading of short duration was applied intravenously (Group 2C), the quantitative values for the S1, S1-2, and S2 loop counts were significantly reversed. Among the superficially located nephrons, the S1-like structures of the initial postglomerular fraction of the proximal tubule were far rarer that the S1-2-like and S2-like cellular structures (see Graph 1A). However, 4 weeks later, these three types of loops at this specific location were equally prevalent in number. In the juxtamedullary nephrons, S1-like structures from the same region were nearly totally eliminated, being replaced primarily by S2-like structures, which were now found to be in two-thirds of the nephrons (see Graph 2B). However, an identical distribution of the loop structures within the segments was found 4 weeks later.

Overall, in terms of the total count, the number of S1 and S1-2 loops in the superficial nephrons was significantly decreased after heavy salt loading of short duration (Group 2C). In contrast, the S2 loop counts were found to have increased significantly (see Graph 2A). These values were subsequently found to normalize in the controls 2 days to 4 weeks after the period of salt loading (see Graph 2A, Group 2D-F). With regard to the same values of total loop counts among the juxtamedullary nephrons, a persistent and significant increase in the number of S2 loops was observed with a heavy salt loading of short duration, a change that continued right up until the last control measurement 4 weeks later. Concomitantly, the number of S1 and S1-2 loops was found to decrease (see Graph 2B).

In the S3 segments from normal, freeze-substituted kidneys, a slight extracellular space could be seen between

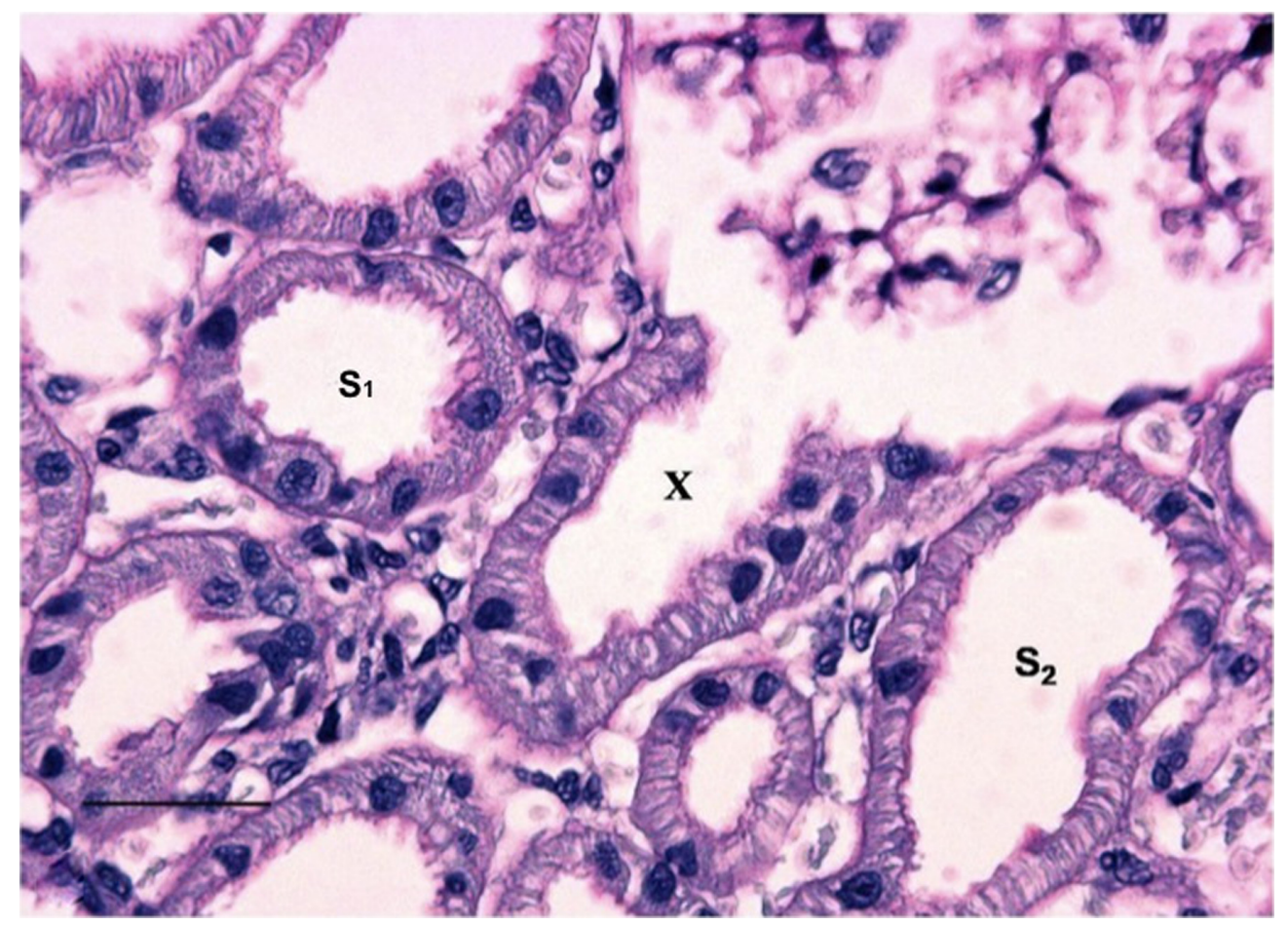

Figure 6 A PAS-stained freeze-substituted kidney, showing the superficial cortex from a rat that had been exposed to a heavy salt-loading of short duration (Group 2 C). Notes: Please note that the proximal tubular loop, descending from the glomerulus (X) has changed in structure from that of a SI to a more typical S2-like cell. Note also, that the luminal diameter, the cellular- and brush border height, and the presence of basolateral spaces are somewhat identical to the structure of the surrounding S2 loops. The change in structure from SI to S2 in the initial postglomerular fraction was more significant among superficial cf. juxtamedullar nephrons (Graph IA and IB). The scale bar represents $25 \mu \mathrm{m}$. 
the lateral cell membranes of some of the $\mathrm{S} 3$ cells (see Figure 7). After exposure to an acute heavy salt loading (Group 2C), a systematic and significant dilatation of the lateral intercellular space was noted (see Figure 8). In the later controls (Groups 2D-F) made 2 days, 2 weeks, and 4 weeks following the acute heavy salt loading, this change in cellular structure was found to normalize (see Figure 9).

The contralateral left kidney was removed from all the animals used in this experiment and formalin fixed (neutral formalin $10 \%$ over a period of 24 hours) according to traditional methodology. After paraffin embedding, sections were stained with hematoxylin and eosin. None of the changes typical of the cryopreparations for the right kidney, with regard to segmentation of the proximal tubule, appearance of intercellular spaces in fractions of the proximal tubule, or nuclear differences or changes in the cells of the proximal tubule, was noted in the left kidneys fixed using traditional methodology. Independent of treatment, eg, "normal", "salt-loaded", or "salt-depleted", the histology sections from the traditionally fixed left kidneys appeared to be microscopically identical.

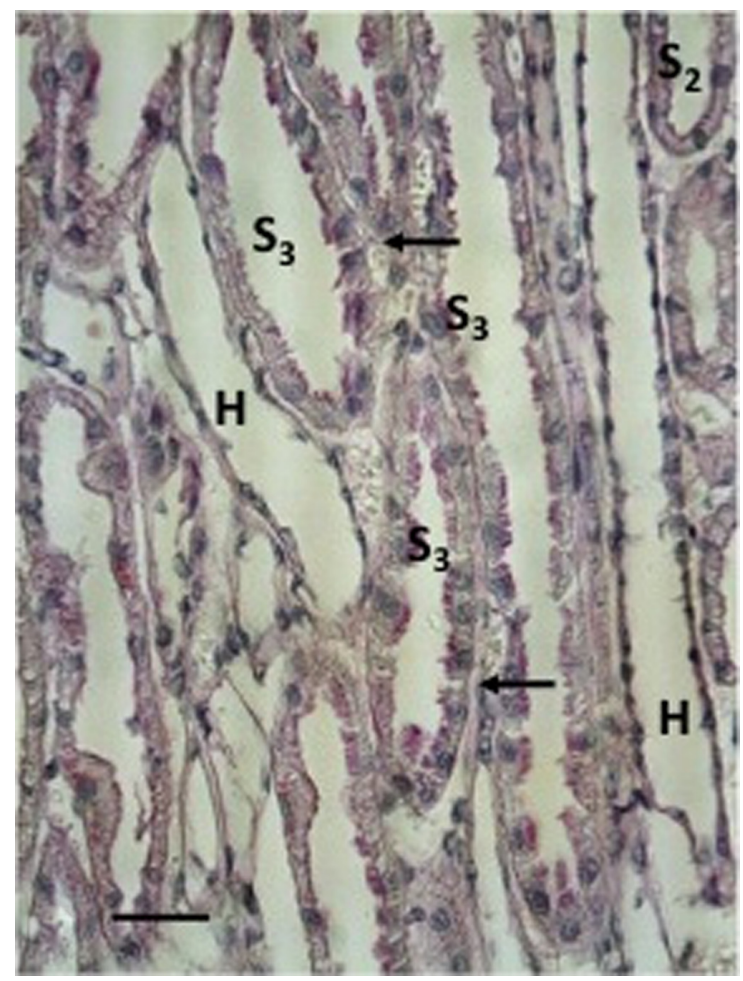

Figure 7 A PAS-stained, freeze-substituted normal kidney (Group IA). Notes: Please note that in the third segment (S3) of the proximal tubule a few open lateral spaces between the cells can be seen (see arrows). $\mathrm{H}$ indicates the ascending branch of the loop of Henle. The scale bar represents $25 \mu \mathrm{m}$.

\section{Discussion}

The present study, which is based on two different cryotechniques, makes possible an entirely new evaluation of the structural background underpinning the considerable water transport that occurs between proximal tubular cells and capillaries (Figures 1-5, 7, and 8). The validity of these newly observed structural details is supported by the fact that the two different cryotechniques used, ie, freeze substitution and special freeze drying, demonstrate identical morphology between similar cell types.

The most important finding of our study with regard to normal kidney function has been the observation of a consistently open basolateral space between the interdigitating S2 segment cells of the proximal tubule, giving rise to an anastomosing, widely open, extracellular compartment for the transport of water (Figures 1-6). A parallel phenomenon of equal importance is an open lateral space between the cells in the $\mathrm{S} 3$ segment (Figure 7). In investigations of the normal rat kidney, in which a cryopreparation was omitted, no dilatation of the basolateral or lateral space between the cells of the proximal tubule was observed. ${ }^{16-19}$ The essential structural differences between the three cell types (S1, S2, and S3)

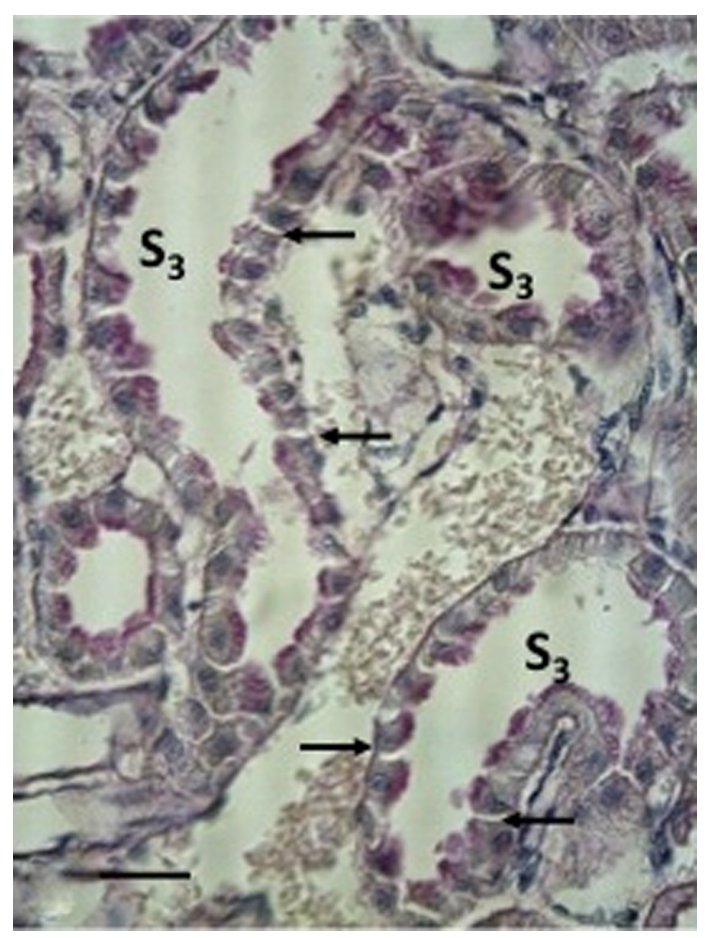

Figure 8 A freeze-substituted and PAS-stained kidney investigated immediately after a heavy salt-loading of short duration (Group 2C).

Notes: Please note, a systematic and significant dilatation of the lateral spaces between the S3 cells can be seen in this section (see arrows). The scale bar represents $25 \mu \mathrm{m}$. 


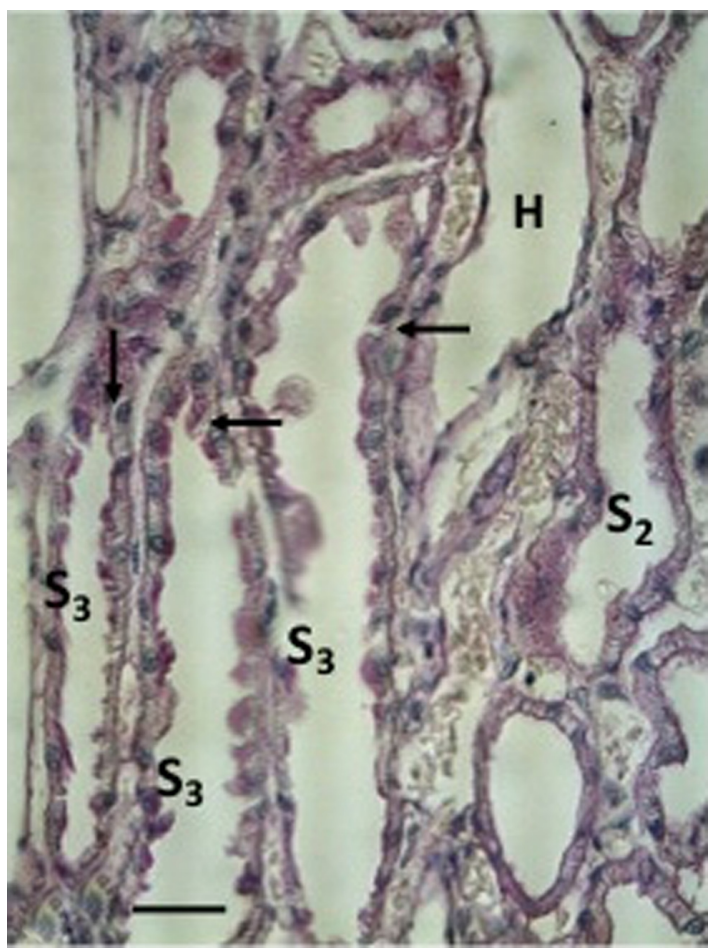

Figure 9 A freeze-substituted and PAS-stained kidney two days following a heavy salt loading of short duration (Group 2F).

Notes: Please note, that between the S3 cells a lesser degree of dilatation of the lateral space can be found associated with fewer cells compared to Figure 8 (see arrows). $\mathrm{H}$ indicates the ascending branch of the loop of Henle. The scale bar represents $25 \mu \mathrm{m}$.

in the proximal tubule, as seen in the cryopreparations of the renal cortex, are illustrated in Figure 10.

The occurrence of intracellular aquaporin channels in the proximal tubular cells, ${ }^{2,3}$ the endings of which were found not only at the basal ends of the cells but also laterally near to the basolateral membranes, is an important finding. Consequently, it follows that the basolateral spaces should form part of the transport route for water from the tubular lumen to the capillary. In the normal kidney, these spaces were dilated in the $\mathrm{S} 2$ segments of the proximal tubules, and to a lesser degree in the S1-2 and S3 segments. This finding raises the question of whether transcellular water transport is of the same order of magnitude in both the S1 and the S2 segment cells, although, at present, no definite conclusions can be drawn. ${ }^{20}$ However, the lack of basolateral spaces identifiable by light microscopy focally in the immediate subcapsular S2 loops (Figure 6) might indicate the functional importance of this structural phenomenon for physiological micropuncture studies at this location.

In the normal rat kidney, the presence of only the typical S1-like cells at the immediate postglomerular region of the proximal tubule is much more frequent in superficial nephrons than in those found in the juxtamedullary nephrons (Graphs 1A, 1B, and 10). However, the opposite is the case
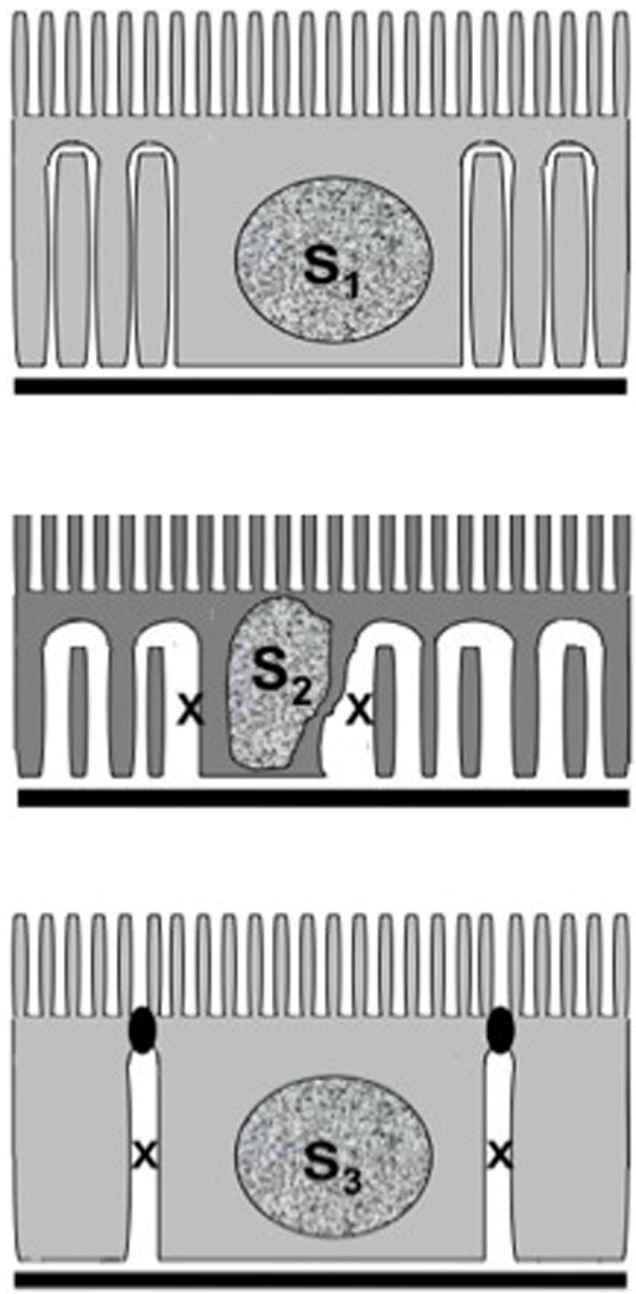

Figure 10 Essential structural features distinguishing the three segments of the proximal tubule: Upper: SI; Middle: S2; and Lower: S3. Only in the S2 and S3 segments is an enlargement of the basolateral or lateral space (marked with an X) seen in cryopreparations of the tissue (compared with Figures 2 and 7-9).

with regard to the S1-2-like fractions at the same location. Superficially, the presence of only S2 cells initially in the proximal tubular loop is nearly nonexistent, although in the juxtamedullary area $30 \%$ of the nephrons are populated solely with the S2 cell type.

The total tubular loop counts for the proximal convoluted tubule in normal kidneys (Graphs 2A and 2B) revealed that the number of $\mathrm{S} 1$ loops is significantly greater in superficial nephrons than in those located in the juxtamedullary region. This is parallel to the occurrence of S1 loops found initially in the postglomerular loops from nephrons located superficially versus those in the juxtamedullary region (Graph 1A and 1B). It can be seen from the same figures that a moderate salt load or salt depletion (Groups 2A and 2B) of 1 month's duration is without significant change initially in the postglomerular loops as well as in the total tubular cell counts (Graph 1A, $1 \mathrm{~B}, 2 \mathrm{~A}$, and 2B). However, when a heavy salt loading of short 
duration was given intravenously over a period of 1 hour, the structure of the tubular cells was seen to change (Group 2C). In the initial postglomerular part of the tubule, the relative proportion of S1-like loops changed from 70\% (normal kidneys) to less than $10 \%$ in the superficial nephrons. In the nephrons of the juxtamedullary region, values for the same location decreased from $17 \%$ to $1 \%$. Correspondingly, significant increases in the number of S2 loops for the same part of the tubule were observed. A similar significant increase in the number of S2 loops was found in the total tubular loop counts (Graphs 2A and 2B). It is thus concluded that some of the typical S1 loops were changed to S2 loops in the total tubular loop counts. This would mean that S1-like and S2-like cells are mutually exchangeable, depending on the functional physiological milieu to which they are exposed. However, the reason for the structure of the S1 cells, having a reduced lumen and an enlarged cell size, is not definitively understood or indeed explained in the literature.

In the freeze-substituted and freeze-dried kidneys, the structure of the S1 cells was found to be identical (Figures 1 and 3). This was also found to be the case for the structure of S2 cells. This is an especially important point when one considers the presence of the dilated basolateral spaces, which were consistently present among these cells in the normal kidney, independent of the type of cryopreparation. Therefore, it is concluded that the open basolateral space between the S2 cells, as well as the open lateral space seen between S3 cells, represents a functional phenomenon rather than a preparative artifact (Figures 1, 2, 3, and 10).

The glomerular filtration rate in normal kidneys is found to be consistently higher in juxtamedullary nephrons than in the superficial ones. ${ }^{21,22}$ Thus, returning to the different functional conditions of S1 cells as compared with S2 cells (eg, glucose transport versus water transport), the higher glomerular filtration rate in the juxtamedullary nephrons may be related to their structural differences. ${ }^{21-23}$ Indeed, this might explain why in the normal kidney the total number of S1 plus S1-2 loops in the proximal convoluted tubule was found to be the same in the superficial and juxtamedullary cortical regions (approximately 40\%, see Graphs 2A and 2B).

Beyond the sparse vascularization of the S2 loops that are found in the immediate subcapsular region, the peritubular capillaries found inside the renal cortex appear very different from the capsular capillaries. This is very important when one considers the hydrostatic as well as oncotic pressure of plasma. In the renal peritubular cortical capillaries, the hydrostatic blood pressure is about $10-15 \mathrm{~mm} \mathrm{Hg}$, whereas the pressure in capillaries outside the kidney is $10-30 \mathrm{~mm} \mathrm{Hg}$. Of course the capsular capillaries generally arise from the lumbar arteries, unlike the peritubular capillaries. Likewise, the oncotic pressure in the peritubular capillaries is in the order of $20-35 \mathrm{~mm} \mathrm{Hg},{ }^{24}$ whereas it is only about $20 \mathrm{~mm} \mathrm{Hg}$ in the capillaries of the systemic circulation. This serves to highlight the fact that the dominant water transport in the renal cortex is due to reabsorption, in contrast with the typical paracapillary water circulation found in the systemic circulation, as described by Starling. ${ }^{25}$ Indeed, this reasoning may go a long way toward providing an explanation for the observation that the lack of open basolateral spaces between the cells of immediately subcapsular S2 loops never exceeds more than $50 \%$ of the total S2 loops.

If one continues this line of reasoning, it seems logical to conclude that the open basolateral space between S2-like cells is the result of functional conditions not only in the tubule but also in the surrounding peritubular capillaries. Moreover, these cellular changes are probably the cause of the angular form of the nuclei in these cells, because they are never found in typical S1 or S3 cells, where all the nuclei have a circular or oval appearance (Figures 1 and 7).

The application of a heavy intravenous salt load of short duration resulted in what appeared to be a conversion of the vast majority of $\mathrm{S} 1$ loops in the immediate postglomerular area from superficial nephrons to S2-like loops (Group 2C, Graphs 1A). This is reflected in the total tubular loop counts where both S1 and S1-2 loops were found to be replaced proportionally with S2-like loops in superficial nephrons, although among the juxtamedullary nephrons only the S1-2 loops showed a significant reduction in number (Graphs 2A and 2B). In both locations, the number of S2-like loops was significantly increased. In order to evaluate the reversibility of these changes, control rats were investigated in which normal feeding was given for a period of 2 days, 2 weeks, and 4 weeks. As can be seen in Graphs 1A and 1B, changes were found to be partly reversible only in the S1 loops of the superficial nephrons. Interestingly, a similar response to normal feeding was not apparent in the juxtamedullary nephrons (see Graphs 2A and 2B). These cellular changes in the superficial nephrons may simply reflect a physiological difference between superficial and juxtamedullary nephrons.

The present data clearly demonstrate that typical S1-like and S2-like cellular structures can be reversed, depending on the biological milieu and cellular response. Moreover, the variations in the tubular segmental cell types show that more permanent changes in distribution of the loops can be seen in both the initial postglomerular loops and later fractions of the convoluted proximal tubule (Graphs 1A, $1 \mathrm{~B}, 2 \mathrm{~A}$, and $2 \mathrm{~B})$. 
It is concluded that the existence of a consistent, open, basolateral, or lateral space between cells from the second and third segment of the proximal tubule in the normal rat kidney is in close agreement with the established concept concerning water transport from tubular cells to peritubular capillaries. Moreover, this study has shown that the distribution of first and second segment cells in tubules is different in superficial nephrons compared with in juxtamedullary nephrons. This finding is consistent with the knowledge that superficial and juxtamedullary nephrons are exposed to different functional conditions. However, perhaps the most exciting finding of our study is that salt loading, in terms of the kidney, results in the transformation of S1 cells to $\mathrm{S} 2$ cells, and vice versa, in the proximal tubule under physiological conditions.

\section{Acknowledgments}

The authors thank Niels Marcher and Paul Mai for their valuable assistance. This work was supported by grants from the Danish Medical Research Council and the Danish Heart Association.

\section{Disclosure}

None of the authors has any competing financial interest in this study. They are all employed in independent research institutions, with no support from sources with a financial interest in this study or its results.

\section{References}

1. Maunsbach AB. Observations on segmentation of the proximal tubule in the rat kidney. Ultrastruct Res. 1966;16:239-245.

2. Nielsen S, Smith BL, Christensen EI, Knepper MA, Nielsen F. Chip 28 water channels are located in constitutively water-permeable segments of the nephron. J Cell Biol. 1993;120:371-383.

3. Maunsbach AB, Marples D, Chin E, et al. Aquaporin-1 water channel expression in human kidney. J Am Soc Nephrol. 1997;8:358-360.

4. Agre P, King LS, Yasui M, et al. Aquaporin water channels - from atomic structure to clinical medicine. J Physiol. 2002;542:3-16.

5. Hanssen O. Early post mortem renal changes studied in mice with one kidney exteriorized. 2. The functional and the early post mortem morphology of the kidney. Acta Pathol Microbiol Scand. 1960;49: 287-320.
6. Hegedüs V, Faarup P. The cortical volume of the normal human kidney. Correlated angiographic and morphological studies. Acta Radiol Diagn. 1972;12:481-496.

7. Faarup P, Saelan H, Ryoe G. Correlation between tubules and capillaries and size of interstitial space in the functioning rat kidney. Acta Pathol Microbiol Scand A. 1971;79:607-616.

8. Tune BM, Burg MB. Glucose transport by proximal renal tubules. Am J Physiol. 1971;221:580-585.

9. Schultz SG, Fuisz RE, Curran PF. Amino acid and sugar transport in rabbit ileum. J Gen Physiol. 1966;49:849-866.

10. Csaky TZ, Esposito G. Osmotic swelling of intestinal epithelial cells during active transport of sugars. Am J Physiol. 1969;217:753-755.

11. Armstrong WMD, Musselman DL, Reitzug HC. Sodium, potassium and water content of isolated bullfrog small intestinal epithelium. Am J Physiol. 1970;219:1023-1026.

12. Steinhausen M, Eisenbach G, Galaske R. A counter-current system of the surface of the renal cortex of rats. Pflügers Arch. 1970;318: 244-258.

13. Faarup P, Holstein-Rathlou NH, Hegedüs V. Structural evidence for counter-current flow in proximal tubules versus peritubular capillaries in the rat kidney. APMIS. 2000;108:779-784.

14. Faarup P, Holstein-Rathlou NH, Nørgaard T, Hegedüs V. Early segmental changes in ischemic acute tubular necrosis of the rat kidney. APMIS. 2004;112:192-200.

15. Drummond GB. Reporting ethical matters in The Journal of Physiology: Standards and advice. J Physiol. 2009;587:713-719.

16. Bulger RE, Lorentz WB Jr, Colindres RE, Gottschalk CW. Morphologic changes in rat renal proximal tubules and their tight functions with increased intraluminal pressure. Lab Invest. 1974;30:136-144.

17. Evan AP, Baker VT, Bengele HH. Zonulae occludentes of the rat nephron under conditions of experimental expansion of blood and/or fluid volume. Anat Rec. 1976;186:139-150

18. Lorenz WB, Lassiter WE, Gottschalk CW. Renal tubular permeability during increased intrarenal pressure. J Clin Invest. 1972;51:484-492.

19. Celsi G, Larsson L, Aperia A. Proximal tubular reabsorption and Na-K-ATPase activity in remnant kidney of young rats. Am J Physiol. 1986;251:F588-F593.

20. Liu FY, Cogan MG, Rector FCJ. Axial heterogeneity in the rat proximal convoluted tubule. II. Osmolality and osmotic water permeability. Am J Physiol. 1984;247:F822-F826.

21. Lameire NH, Lifschitz MD, Stein JH. Heterogeneity of nephron function. Ann Rev Physiol. 1977;39:159-184.

22. Kritz W, Kaissling B. Structural organization of the mammalian kidney. In: Seldin DW, Giebisch G, editors. The Kidney, Physiology and Pathophysiology. New York, NY: Raven Press; 1985.

23. Treeck B, Aukland K. Effect of L-NAME on glomerular filtration rate in deep and superficial layers of rat kidneys. Am J Physiol. 1997; 272(3 Pt 2):F312-F318

24. Gottschalk CW, Mylle M. Micropuncture study of pressures in proximal tubules and peritubular capillaries of the rat kidney and their relation to ureteral and renal venous pressures. Am J Physiol. 1956;185:430-439.

25. Starling EH. On the absorption of fluids from the connective tissue spaces. J Physiol (Lond). 1896;19:312-326.

\section{Publish your work in this journal}

The International Journal of Nephrology and Renovascular Disease is an international, peer-reviewed open-access journal focusing on the pathophysiology of the kidney and vascular supply. Epidemiology, screening, diagnosis, and treatment interventions are covered as well as basic science, biochemical and immunological studies. The journal welcomes original research, clinical studies, reviews \& evaluations, expert opinion and commentary, case reports and extended reports. The manuscript management system is completely online and includes a very quick and fair peer-review system, which is all easy to use. Visit http://www.dovepress. com/testimonials.php to read real quotes from published authors. 